\title{
Insuffisance Rénale Aigue Post-Opératoire Au Centre Hospitalier Universitaire Et Départemental Du Borgou : Fréquence Et Facteurs De Risques Associés
}

\begin{abstract}
Ahoui Séraphin
Faculté de Médecine (FM) de l’Université de Parakou ; Service de néphrologie, Centre Hospitalier Universitaire et Départemental du Borgou Parakou Bénin.

Hodonou Montcho Adrien

Allode Salako Alexandre

Faculté de Médecine de l’Université de Parakou ; Service de chirurgie générale, Centre Hospitalier Universitaire et Départemental du Borgou Parakou Bénin.
\end{abstract}

\section{Tchaou Blaise}

Faculté de Médecine de l'Université de Parakou ; Service d d'accueil Urgence et Reanimation Centre Hospitalier Universitaire et Départemental du Borgou Parakou Bénin.

Vigan Jacques

Faculté des Sciences de Santé de l'Université d'Abomey Calavi ; Clinique Universtaire de Néphrologie et d'Hémodialyse Centre National Hospitalier Universitaire Cotonou Bénin.

\section{Agbidinoukoun Roméo}

Faculté de Médecine de l’Université de Parakou Bénin

Salifou Kabibou

Faculté de Médecine de l’Université de Parakou ; Service de gynécologie obstétrique Centre Hospitalier Universitaire et Départemental du

Borgou Parakou Bénin.

doi: 10.19044/esj.2016.v12n33p188 URL:http://dx.doi.org/10.19044/esj.2016.v12n33p188

\begin{abstract}
Introduction: Acute renal failure (ARF) post-operative is a specific form of acute deficiencies causing multiple declining factors.

Objective: This survey aims to study the incidence and risk factors associated with acute renal failure (ARF) in post-operative surgical intensive care units to University Hospital of Borgou (UH-B): during 2015.

Patients and Methods this study is a cross sectional, descriptive and analytical ones with the prospective data collection from March 1st to
\end{abstract}


August 31th, 2015. The research has involved all patients admitted to the operating room for surgery and motherhood whatever reason and then transferred respectively to the intensive care areas at UH-B. The postoperative ARF has been investigated inner patients following inclusion criteria and classification according to RIFLE score. The socio-demographic, clinical and biological variables monitoring, even support and evolution are experimented. A questionnaire is designed for data collection. Data are analyzed by Epi-Info means with 5\% of significance level.

Results: 130 patients are registered. The mean age is $27.68 \pm 12.87$ years. The sex ratio is 0.66 . The frequency of post-operative ARF reaches $12.31 \%$. The associated risk factors are: hypertension $(\mathrm{p}=0.0018)$, diabetes $(\mathrm{p}=$ $0.002)$, heart failure $(p=0.0104)$, severe sepsis $(p=0.006)$ hypovolemic shock $(\mathrm{p}=0.002)$, ASA class $\geq 3(\mathrm{p}=0.0014)$, preeclampsia-eclampsia $(\mathrm{p}=$ $0.012)$, the Altémier class classification $\geq 3(\mathrm{p}=0.0164)$, a pathological urinary sediment like a proteinuria $(\mathrm{p}=0.006)$, haematuria $(\mathrm{p}=0.001)$ and nitrituria ( $\mathrm{p}=0.007)$. Consequently, three (03) subjects out of sixteen (16) have died (18.75\%).

Conclusion: The post-operative ARF is a reality in University Hospital Borgou, with a higher mortality rate. Thus, the prevention strategy is the best treatment through the screening and monitoring promotion towards risk factors.

Keywords: Post-operative ARF, frequency, risk factors, University Hospital Borgou

\section{Resume}

Introduction : L'insuffisance rénale aigue (IRA) post-opératoire est une forme particulière des insuffisances aigues avec plusieurs facteurs déclenchants. L'objectif était d'étudier la fréquence et les facteurs de risque associés à l'insuffisance rénale aigue (IRA) post-opératoire dans les services de réanimation chirurgicale du CHUD-Borgou en 2015.

Patients et Méthodes Il s'agissait d'une étude transversale, descriptive et analytique avec recueil prospectif des données du $1^{\mathrm{er}}$ Mars au 31 Août 2015. Tous les patients opérés (de la chirurgie et de la maternité) et transférés dans les secteurs de la réanimation chirurgicale étaient concernés. Le score de RIFLE a permis de diagnostiquer l'IRA post opératoire. Les variables socio démographiques, clinico-biologiques celles de prise en charge de suivi et d'évolution ont été étudiées. Un questionnaire a été établi pour la collecte des données. Les données collectées ont été analysées avec le logiciel Epi-Info 7.1.1.14 avec un seuil de significativité de 5\%.

Résultats : Au total, 130 patients ont été enregistrés. L’âge moyen était de $27,68 \pm 12,87$ ans. La sex-ratio était de 0,66. La fréquence de l'IRA post 
opératoire était de 12,31\%. Les facteurs de risque associés étaient : l'hypertension artérielle $(p=0,0018)$,le diabète $(p=0,002)$, l'insuffisance cardiaque $(\mathrm{p}=0,0104)$, le sepsis sévère $(\mathrm{p}=0,006)$, l' état de choc hypovolémique $(\mathrm{p}=0,002)$, la classe $\mathrm{ASA} \geq 3 \quad(\mathrm{p}=0,0014)$, la pré éclampsie-éclampsie $(p=0,012)$, la classification $d$ 'Altémier $\geq$ classe $3(p=0,0164)$, un sédiment urinaire pathologique comme une albuminurie $(\mathrm{p}=0,006)$, hématurie $(\mathrm{p}=0,001)$ et une nitriturie $(\mathrm{p}=0,007)$. La létalité était de trois sur 16 soit $18,75 \%$

Conclusion : L'IRA post opératoire est une réalité au CHUD Borgou avec un taux de mortalité élevé. La prévention reste le meilleur traitement et passe par le dépistage et le suivi des facteurs de risque.

Mots clés : IRA postopératoire, facteurs de risque, réanimation, CHUDBorgou

\section{Introduction}

L’incidence élevée de l’insuffisance rénale aiguë (IRA) postopératoire est liée à l'importance des facteurs de risque et à l'insuffisance de leur dépistage et prise en charge [Deman et al. 2004]

En Belgique en 2004, l’IRA post opératoire constituait la deuxième cause des IRA acquises à l'hôpital avec une fréquence de 18 à 47 \% [Deman et al. 2004]. En France l'incidence de l’IRA post-opératoire était évaluée entre 0,1 et $2 \%$ toutes chirurgies confondues [Kellerman , 1994] En Afrique, la fréquence des IRA postopératoires varie de 13\% à 28\% [Assouto et al., 2009, Mbiandoun Ngatcha, 2013]. Au Bénin, Assouto et al. en 2009 dans le domaine de la chirurgie digestive avaient retrouvé une incidence de l'IRA post opératoire évaluée à 1,8\%, avec la péritonite aiguë qui était l’indication chirurgicale à risque la plus représentée (27,4\%). [Assouto et al., 2009]. Au Nord du Benin, aucune étude n’a été réalisée pour évaluer la fréquence et les facteurs de risque associés à l'IRA post opératoire.

La présente étude a été initiée au Centre Hospitalier Universitaire et Départemental du Borgou, (CHUD-B), afin d'évaluer la fréquence et les facteurs de risque associés à l'IRA post opératoire.

\section{Cadre, patients et méthode d'étude}

L’étude s’est déroulée dans les secteurs de réanimation chirurgicale du Centre Hospitalier Universitaire et Départemental du Borgou (CHUD-B) à Parakou, seule ville septentrionale du Bénin située à 400 kilomètres de Cotonou et disposant d'un service de réanimation polyvalente avec un médecin anesthésiste-réanimateur et un médecin néphrologue. L’étude était observationnelle, transversale, descriptive et analytique avec recueil 
prospectif des données sur une période de 6mois, allant du $1^{\mathrm{er}}$ Mars 2015 au 31 Août 2015.

Etaient inclus dans l'étude tout patient admis aux blocs opératoires du CHUD-B avec la notion d'une fonction rénale antérieurement normale ayant séjourné plus de 24 h en réanimation. Les prélèvements sanguins étaient réalisés au plus tard 6 heures avant l'acte opératoire et puis en $24 \mathrm{~h}$ et au $7^{\text {ème }}$ jour en période post opératoire.

L’insuffisance rénale aigue en postopératoire a été retenue sur l'augmentation de l'urémie au-delà de 0,45 g/l, de la créatininémie supérieure à $12 \mathrm{mg} / \mathrm{l}$ chez la femme enceinte, $13 \mathrm{mg} / \mathrm{l}$ chez la femme non enceinte et $14 \mathrm{mg} / \mathrm{l}$ chez l'homme et par le score de RIFLE (Risk, Injury, Failure, Lost and End-stage). Ce score tient compte du Débit de Filtration Glomérulaire (DFG) calculé selon la formule de Cockcroft et Gault. [Agence nationale d'accréditation et d'évaluation en santé(ANAES). Recommandations pour la pratique clinique, 2002]

(DFG $=(140$ - âge) $X$ Poids $X \mathrm{k} / 7,2 \mathrm{X}$ créatininémie) $\mathrm{k}=1$ chez l'homme et 0,85 chez la femme Poids en $\mathrm{Kg}$, âge en années, créatinémie en mg/l. [Agence nationale d'accréditation et d'évaluation en santé(ANAES). Recommandations pour la pratique clinique, 2002]

Un recrutement exhaustif de tous les patients remplissant les critères d'inclusion et qui étaient admis aux blocs du CHUD-B, et transférés dans lesdits secteurs de la réanimation pendant la période d'étude a été réalisé.

N'étaient pas inclus tous les patients diagnostiqués insuffisance rénale à l'admission en préopératoire, les opérés en ambulatoire et ayant séjourné moins de 24 h en réanimation.

L’échantillonnage était exhaustif en série continue

Une IRA post-opératoire était considérée précoce si elle survient avant $48 \mathrm{~h}$ et tardive entre la $48^{\text {ème }}$ heure et le $7^{\text {ème }}$ jour. La variable dépendante était l'existence d'une insuffisance rénale aiguë post-opératoire. Pour étudier les facteurs de risque de l'IRA post-opératoire les caractéristiques sociodémographiques (âge, sexe, IMC), cliniques (antécédents de maladie, sédiment urinaire à la bandelette urinaire, indication opératoire et intervention chirurgicale), biologiques (CRP, NFS ionogramme sanguin) le score ASA permettant d'évaluer l'aptitude du patient à supporter une intervention chirurgicale ou pas et la classification d'Altémeier

- Les données collectées ont été traitées grâce logiciel Epi info version 7.1.1.14. Le seuil de significativité des tests est fixé à $p<0,05$.

\section{Résultats}

Au total, 202 patients opérés ont été colligés durant la période d'étude. Parmi eux 130 patients avaient une fonction rénale normale à l'admission. 


\section{Fréquence de l'IRA post opératoire}

Parmi les 130 patients enquêtés, 16 avaient présenté une IRA post opératoire dans les 48 premières heures suivant l'intervention soit une fréquence de $12,31 \%$.

L'IRA était persistante après sept jours chez 8 patients soit une fréquence de $6,15 \%$

\section{Caractéristiques socio-démographiques}

L'âge moyen des patients était de 27,68 \pm 12,87 ans (extrêmes allant de 1 an à 76 ans). La tranche d'âge la plus représentée était celle de 15 à 30 ans (59,70\%). Sur les 130 patients, 78 étaient des femmes (60\%), soit une sex-ratio de 0,66 . Ces résultats sont présentés dans le tableau $\mathrm{N}^{\circ} \mathrm{I}$

\section{Caractéristiques clinique des patients}

Les antécédents médicaux d'HTA, d'insuffisance cardiaque et du diabète ont été notés respectivement chez $12,96 \%$ 4,56\% et 3,49\% des cas.

A l'admission, l'albuminurie, l'hématurie, la leucocyturie et la nitriturie était respectivement positive chez 12,94\%, 5,97\% 9,02 \% et $8,46 \%$ des patients. On a noté une glucosurie chez $0,99 \%$ Le sédiment urinaire n'a pas été modifié 24 heures après l'intervention chirurgicale.

\section{Indication opératoire}

Les indications opératoires les plus retrouvées chez les patients opérées de notre série était: la souffrance fœtale aigue $(24,86 \%)$, la péritonite (20,82\%), la pré éclampsie et l'éclampsie (16,86\%), l’appendicite aigue $(14,88 \%)$, la disproportion foeto-pelvienne (9,90\%), la pelvipéritonite $(6,74 \%)$ et les fractures de membres (5,94\%).

Le score ASA

La classe ASA 3 était le plus représenté avec 34,38\%. Pourtant les classes ASA 1 et ASA 2 étaient respectivement retrouvées dans 26,56 et $25,00 \%$ des cas. La classe ASA 4 représentait 13,02\% et ASA 5 1,04\%

\section{Traitement chirurgicale}

La césarienne était pratiquée chez 37,69\% des patients. La chirurgie viscérale et la neurochirurgie étaient pratiquées respectivement chez 42,31\% et 7,38\% des patients. Quant à la traumatologie et l'urologie respectivement 9,33\% et 3,33\% des patients en avaient bénéficié.

La chirurgie était pratiquée en urgence chez 54,00\% des patients. 


\section{La classification d'Altémeier}

La chirurgie propre et celle propre contaminée étaient respectivement retrouvée dans 30,69 et 45,54\%. La chirurgie contaminée et la chirurgie sale étaient respectivement de $14,85 \%$ et de $8,81 . \%$.

\section{Type de ventilation, d'anesthésie, durée de l'intervention et les positions per opératoire}

L’anesthésie locorégionale et l'anesthésie générale étaient pratiquée respectivement chez $70 \%$ et $30,00 \%$ des patients. La ventilation à la lunette était pratiquée pour $80,66 \%$ et celle au masque chez 19,34\%

Le décubitus dorsal et le décubitus ventral comme position per opératoire étaient adoptés respectivement pour $90,00 \%$ et $10,00 \%$ des patients.

La durée de l'intervention chirurgicale était inférieure à 120 minutes pour $68,66 \%$ patients et elle était supérieure à 240 minutes pour $6,00 \%$. La durée comprise ente entre 120 et 359 minutes était retrouvée chez 24,66\%

\section{Perte Sanguine}

La quantité de perte sanguine comprise entre 200-400cc a été retrouvée chez $82,18 \%$ et celle entre 100-200cc chez 17,82\% des patients.

\section{Défaillances viscérales}

Sur les 150 patients admis, 41 soit 27,33\% avaient présenté des defaillances viscérales.

Comme défaillances viscérales il a été retrouvé 36,59\% de sepsis sévères, 29,26\% d'état de choc hypovolémique, 24,39\% de coma. Les défaillances respiratoires et cardiaques occupaient chacune $4,88 \%$ des cas.

\section{Evolution des cas d'IRA post opératoire selon le score de RIFLE}

Le score RIFLE était classé Risk (R), Injury (I) et Failure (F) 48 heures après l'opération respectivement chez neuf $(56,25 \%)$, six $(37,5 \%)$ et un $(6,25 \%)$ patients. Au $7^{\text {ème }}$ jour de la période post opératoire, l'évolution du score était retrouvée pour R, I et F respectivement pour quatre (25\%) et trois (18,35\%) et zéro des patients. Trois patients étaient décédés sur les 16 ayant souffert de l'IRA post-opératoire soit une de mortalité de 18,75\%. Une récupération totale a été observée avant trois mois chez les 13 survivants soit $81,25 \%$. 


\section{Facteurs associés à la survenue de l'IRA post opératoire au CHUD Borgou en 2015}

La survenue de l'IRA postopératoire était statistiquement associée aux antécédents d'hypertension artérielle $(p=0,0018)$, diabète $(p=0,002)$ et insuffisance cardiaque $(\mathrm{p}<0,0104)$

La classe ASA 3 était statistiquement associée à la survenue d'une L’IRA post opératoire ( $\mathrm{p}=0,0014)$.

L’IRA post opératoire était significativement plus fréquente chez les patients avec une albuminurie positive $(\mathrm{p}=0,006)$ hématurie positive $(\mathrm{p}=$ $0,001)$ et une nitriturie positive $(p=0,007)$.

L'IRA post opératoire était significativement associée à la valeur préopératoire de la CRP $(\mathrm{p}=0,008)$

La fréquence des IRA variait significativement selon la classification d’Altémier ( $\mathrm{p}=0,0164)$

Par rapport à l’indication chirurgicale la pré éclampsie et l’éclampsie étaient liées à la survenue de l'IRA post- opératoire.

Les défaillances viscérales étaient associées à la survenue de l’IRA post opératoire. Il s'est agi de sepsis sévères $(p=0,006)$, l’état de choc hypovolémique $(p=0,002)$, la défaillance respiratoire $(p=0,013)$ et la défaillance cardiaque $(p=0,013)$. Le nombre moyen de défaillances associées par patient est de 1,25. Le tableau II résume la relation entre la survenue de l'IRA post opératoire et les facteurs associés.

\section{Discussion}

\section{Fréquence de l'IRA post-opératoire}

Dans notre étude la fréquence de l'IRA post opératoire était de 12,31\%. Ce résultat est proche de celui de Lengani et al. [Lengani et al. 2006] au Burkina en 2006 (13,2\%), mais 10 fois supérieur à celui Deman et al.[Deman et al., 2004] en Belgique en 2004 qui ont rapporté une fréquence de 1,2\%. Le dépistage précoce des facteurs de risque ainsi que leur suivi rigoureux en période péri-opératoire pourrait contribuer à réduire l'incidence telle que observée dans cette étude.

\section{Facteurs associés à la survenue de l'IRA post-opératoire au CHUD-B en 2015}

La survenue de l'IRA postopératoire était statistiquement associée aux antécédents morbides [Brivet et al. 1996, de Mendonca et al. 2000, Guerin et al., 2000]. Dans notre étude, l'hypertension artérielle, le diabète et l'insuffisance cardiaque étaient des facteurs de risque de l'IRA postopératoire. Nos résultats sont conformes à ceux de la littérature.

L’IRA post opératoire était significativement plus fréquente chez les patients avec une albuminurie positive, hématurie positive et une nitriturie 
positive. L’hémoglobine éliminée dans les urines entraine une toxicité tubulaire par précipitation dans la lumière tubulaire en formant des agrégats, ce qui conditionne l'obstruction tubulaire favorisée par un pH urinaire acide. La nitriturie est positive dans les cas d'infection urinaire.

La défaillance multi viscérale préopératoire constatée dans notre étude comme le rendent compte le score ASA et la classe Altémeir de chirurgie est une constance dans beaucoup d'études. [Brivet et al., 1996, Guérin et al.,2000]. En effet l'insuffisance cardiaque; défaillance la plus retrouvée dans notre série, est de cause hypovolémique. Sa persistance engendre une hypo perfusion rénale à l'origine de l'IRA fonctionnelle hémodynamique. Quant au sepsis sévère deux mécanismes physiopathologiques pourraient expliquer la genèse de l'IRA. En effet certains germes déterminant les états infectieux ont un tropisme rénal provoquant une altération directe des néphrons responsable d'une IRA organique. L’hypovolémie relative associée à l’acidose métabolique des états infectieux constitue le deuxième mécanisme des I.RA post-opératoires.

La prééclampsie-éclampsie était significativement associée à la survenue de l'IRA post-opératoire $(=0,0123)$ Jonard et al. ( 2014) en France ont retrouvé des données similaires à la nôtre.[Jonard M, 2014]. La Pré éclampsie-éclampsie entraine une dysfonction endothéliale, un état de vasoconstriction, une hypovolémie et une majoration de l'état pro coagulant.

L’IRA post-opératoire était survenue dans un délai inférieur à 2 jours. Au Bénin, Assouto et al. [Assouto et al. 2009] et Mbiandoun Ngatcha [Mbiandoun Ngatcha, 2013] au Mali ont rapporté des données similaires à notre étude. Dans les 48 premières heures post opératoires, le remplissage vasculaire devrait être adapté en fonction, des pertes d'humeur et d'organes (pièces opératoires), de la diurèse horaire, et de l’état septique.

\section{Conclusion}

En conclusion il ressort de notre étude que l'IRA post-opératoire est fréquente chez les patients au CHUD/B. Plusieurs facteurs expliquent la survenue de l'IRA post-opératoire. La prévention de ces facteurs de risque permettra d'éviter les complications rénales post-opératoires.

\section{References:}

1. Agence nationale d'accréditation et d'évaluation en santé. Diagnostic de l'insuffisance rénale chronique chez l'adulte. Recommandations pour la pratique clinique. Paris: ANAES; 2002.

2. Assouto P, Tchaou B, Kangni N, Padonou J.L, Lokossou T, Djiconkpode I, Aguemon A et al. Évolution postopératoire précoce en chirurgie digestive en milieu tropicale. Med Trop. 2009;(69):477479. 
3. Brivet FG, Kleinknnecht DJ, Loirat P, Landais PJ. Acute renal Failure in the medical intensive care units - causes, out come and prognostic Factors of Hospital mortality: a prospective, multicenter study. Crit Care Med. 1996 Feb;24(2):192-8

4. Deman A, Hoste E, Van Biesen W, Vanholder R. Insuffisance Rénale Aiguë Postopératoire : Épidémiologie, Causes, Pronostic Et Traitement; Flammarion médecine-sciences — actualités néphrologiques Belgique 2004 ; 28 (3) : 255 (229).

5. Guerin C, Girard R, Selli JM, Perdrix JP, Ayzac L. Initial versus delayed acute renal failure in the intensive care unit. A multicenter prospective epidemiological study. Rhone-Alpes Area Study Group on Acute Renal Failure. Am J Respir Crit Care Med 2000;161:872879

6. Jonard M, Ducloy-Bouthors AS, Boyle E, Aucourt M, Gasan G, Jourdain M, et al., Postpartum acute renal failure: a multicenter study of risk factors in patients admitted to ICU, Ann Intensive Care. 2014; 4: 36

7. Kellerman PS. Perioperative care of the renal patient. Arch Intern Med. 1994 Aug 8;154(15):1674-88.

8. Lengani AD Kargougou A. Insuffisance rénale aigue au Burkina Faso Nephr \& therap. 2010 ; (6) : 28-34.

9. Mbiandoun Ngatcha GS. Complications post-opératoire en milieu de réanimation : Profil épidémiologique et clinique ;Thèse de médecine Bamako Mali ; 2013 Bamako129-6

10. de Mendonca A, Vincent JL, Suter PM, Moreno R, Dearden NM, Antonelli M. et al. Acute Renal Failure in the ICU : Risk Factors and outcome evaluated by the SOFA score. Intensive Care Med. 2000 Jul;26(7):915-21

\begin{tabular}{cccccccc}
\hline \multirow{2}{*}{ Variables } & \multicolumn{7}{c}{ IRA post opératoire } \\
& Préopératoire & \multicolumn{2}{c}{ (24h après) } & (7 jours après) & \multirow{2}{*}{$p$} \\
\cline { 2 - 6 } & $\mathrm{n}$ & $\%$ & $\mathrm{~N}$ & $\%$ & $\mathrm{~N}$ & $\%$ & \\
\hline Créatininémie (mgl// & & & & & & & 0,0007 \\
l) & & & & & & & \\
$3,02-12,00$ & 130 & 100,00 & 114 & 87,69 & 122 & 9385 & \\
$12,01-98,76$ & - & - & 16 & 12,31 & 8 & 06,15 & \\
DFG (ml/mn) & & & & & & & 0,0092 \\
$<60$ & - & - & 08 & 06,15 & 05 & 03,85 & \\
$60-120$ & 16 & 12,00 & 21 & 16,15 & 20 & 15,38 & \\
$\geq 121$ & 114 & 86,66 & 101 & 77,30 & 125 & 80,77 & \\
\hline
\end{tabular}

Tableau I : Fréquence des Insuffisances rénales aigue post-opératoires au Centre Hospitalier Universitaire et Départemental du Borgou en 2015 
Tableau II : Relation entre Insuffisance rénale aigue post-opératoire et les facteurs de risque au Centre Hospitalier Universitaire et Départemental du Borgou en 2015.

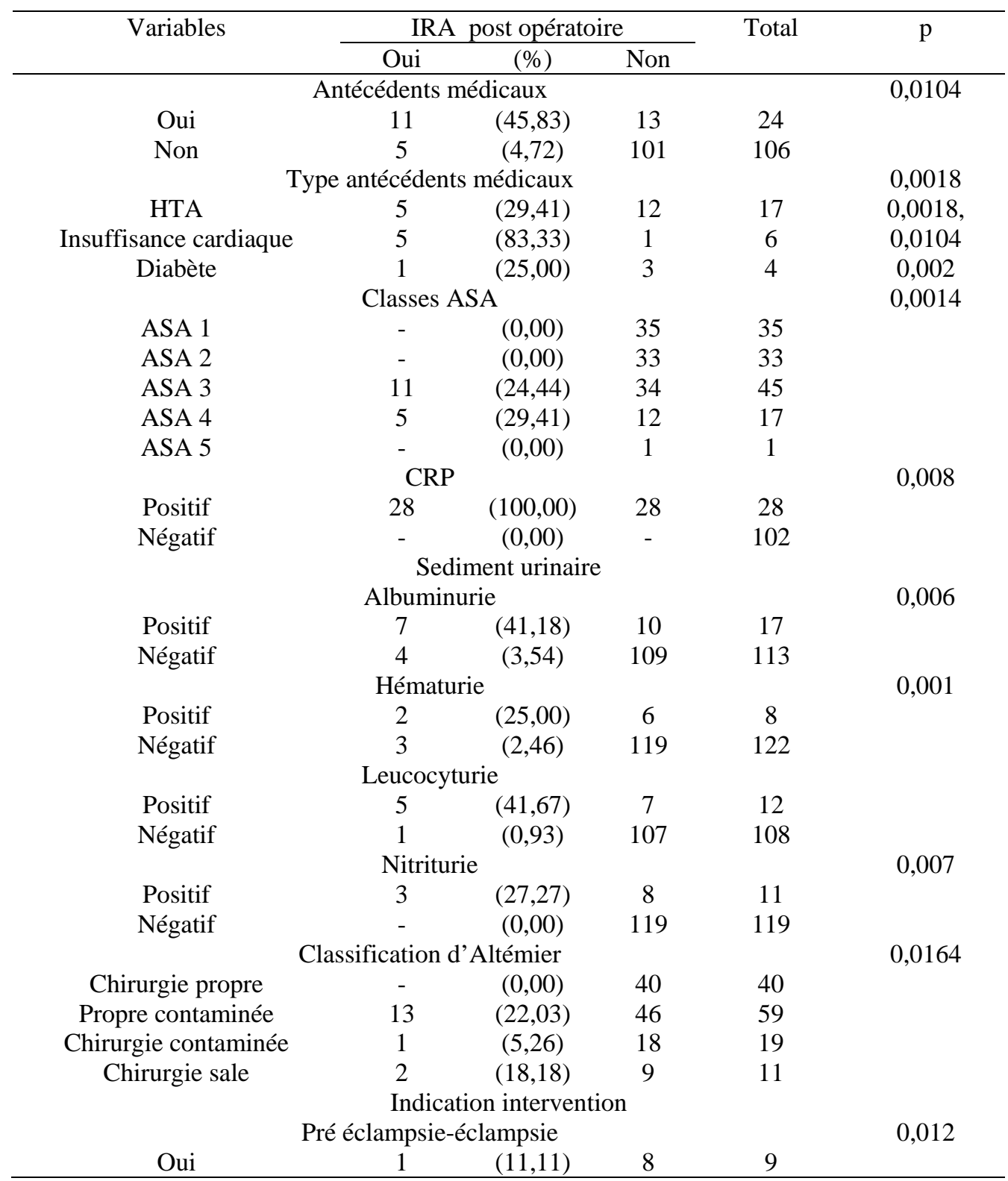

